\title{
A numerical method for two-dimensional Hammerstein integral equations
}

\author{
Sanda Micula
}

Dedicated to Professor Gheorghe Coman on the occasion of his 85th anniversary.

\begin{abstract}
In this paper we investigate a collocation method for the approximate solution of Hammerstein integral equations in two dimensions. As in [8], collocation is applied to a reformulation of the equation in a new unknown, thus reducing the computational cost and simplifying the implementation. We start with a special type of piecewise linear interpolation over triangles for a reformulation of the equation. This leads to a numerical integration scheme that can then be extended to any bounded domain in $\mathbb{R}^{2}$, which is used in collocation. We analyze and prove the convergence of the method and give error estimates. As the quadrature formula has a higher degree of precision than expected with linear interpolation, the resulting collocation method is superconvergent, thus requiring fewer iterations for a desired accuracy. We show the applicability of the proposed scheme on numerical examples and discuss future research ideas in this area.
\end{abstract}

Mathematics Subject Classification (2010): 41A15, 45B05, 47G10, 65D07, 65R20.

Keywords: Hammerstein integral equations, spline collocation, interpolation.

\section{Introduction}

Integral equations are a special topic in Applied Mathematics, as they are an important tool for modeling many applications in fields ranging from engineering, computer graphics to astrophysics, chemistry, quantum mechanics and more (see [13]). They also arise in reformulations of initial and boundary value problems for ordinary and partial differential equations.

Having such a wide variety of applications, they have been studied extensively, especially form the approximation perspective. Numerical solutions have been obtained using moving least squares [5], Adomian decomposition, [4], kernel methods [6], collocation $[8,3,10,7]$, Galerkin and Nyström methods [9]. Also, good results were 
obtained using wavelets $[11,12]$ and other iterative methods [1]. For more details on approximating methods for integral equations, see [2].

In this paper, we consider the following integral equation of Hammerstein type

$$
u(x)=\int_{D} k(x, y) g(y, u(y)) d y+f(x), x \in D \subset \mathbb{R}^{2},
$$

with a smooth kernel $k$ and $g: D \times \mathbb{R} \rightarrow \mathbb{R}$ a continuous nonlinear function. Later, other assumptions will be made on $k, g$ and $f$.

As in [8], collocation is applied to a reformulation of the equation in a new unknown, thus reducing the computational cost and simplifying the implementation. For the new integral equation, we define a collocation scheme based on linear interpolation and show that at the collocation nodes, it converges faster than over the entire domain, thus requiring fewer iterations for a given accuracy. These two aspects make this method much more efficient from the computational point of view.

In operator form, we write (1.1) as

$$
u=K u+f
$$

where

$$
(K u)(x)=\int_{D} k(x, y) g(y, u(y)) d y .
$$

Following the ideas in [8], we reformulate (1.1) for the new unknown

$$
v(y):=g(y, u(y)) \text {. }
$$

By (1.1), $v$ must satisfy the equation

$$
v(x)=g\left(x, \int_{D} k(x, y) v(y) d y+f(x)\right), x \in D
$$

and $u$ is given by

$$
u(x)=\int_{D} k(x, y) v(y) d y+f(x), x \in D .
$$

We define a collocation scheme for $v$ in equation (1.4), which will be then used to find an approximate solution of (1.5).

We are interested in finding a numerical solution of equation (1.1), which approximates the exact solution, assumed to exist. To this end, we work under the following assumptions:

(A1) The equation (1.3) has an isolated solution $u^{*}$ with non-zero index, which is assumed to be smooth enough;

(A2) The integral operator $K: C(D) \rightarrow C(D)$ defined by (1.3) is completely continuous;

(A3) The derivative $g_{u}(y, u)$ exists and is continuous on $D \times \mathbb{R}$;

(A4) The function $f \in C(D)$. 
The ideas and results described in this paper work for any closed bounded domain $D \subset \mathbb{R}^{2}$ that can be triangulated in a smooth way. For simplicity, we restrict the discussion to the case of a rectangular region $D=[a, b] \times[c, d]$.

The rest of the paper is organized as follows: in Section 2, we define a collocation scheme for equation (1.4), based on a special type of linear interpolation on a triangular region. We prove the convergence and give error estimates (for both $u$ nd $v$ ) in Section 3. Section 4 shows the applicability of the proposed method to numerical examples, where the theoretical error bounds are confirmed by the numerical results. We draw some important conclusions and discuss future research ideas in Section 5.

\section{Numerical method}

\subsection{Preliminaries for collocation}

Let us recall the collocation method in the general framework of projection methods. Consider a set of nodes $\left\{x_{1}, \ldots, x_{n}\right\} \subset D$ and let $\left\{l_{1}, \ldots, l_{n}\right\}$ be a set of functions defined on $D$ such that

$$
l_{j}\left(x_{i}\right)=\delta_{i j}, 1 \leq i, j \leq n .
$$

Denote by $D_{n}=\operatorname{span}\left\{l_{1}, \ldots, l_{n}\right\}$ and define the interpolatory projection operator $P_{n}: D \rightarrow D_{n}$ by

$$
\left(P_{n} u\right)(x)=\sum_{j=1}^{n} u\left(x_{j}\right) l_{j}(x), x \in D .
$$

Then $P_{n}: C(D) \rightarrow C(D)$ is a linear operator (see e.g. [2]) and its norm is given by

$$
\left\|P_{n}\right\|=\sup _{x \in D} \sum_{j=1}^{n}\left|l_{j}(x)\right| \text {. }
$$

We will assume that $\left\|P_{n}\right\|<\infty$ and that

$$
\lim _{n \rightarrow \infty}\left\|u-P_{n} u\right\|=0, \text { for all } u \in C(D) .
$$

Let $v^{*}$ be the solution of (1.4) corresponding to $u^{*}$. Using $P_{n}$, we define an approximation of $v^{*}$ by

$$
v_{n}(x)=P_{n} v(x)=\sum_{j=1}^{n} v_{n}\left(x_{j}\right) l_{j}(x) .
$$

The values $\left\{v_{n}\left(x_{j}\right)\right\}_{j=1}^{n}$ are determined by forcing equation (1.4) to be true at the collocation points. This leads to the system

$$
v_{n}\left(x_{i}\right)=g\left(x_{i}, \sum_{j=1}^{n} v_{n}\left(x_{j}\right) \int_{D} k\left(x_{i}, y\right) l_{j}(y) d y+f\left(x_{i}\right)\right), i=1, \ldots, m,
$$

or

$$
\sum_{j=1}^{n} v_{n}\left(x_{j}\right) l_{j}\left(x_{i}\right)=g\left(x_{i}, \sum_{j=1}^{n} v_{n}\left(x_{j}\right) \int_{D} k\left(x_{i}, y\right) l_{j}(y) d y+f\left(x_{i}\right)\right) .
$$


It is worth mentioning that the integrals on the right hand side only have to be evaluated once, not at every iteration, since they are dependent only on the basis functions, not on $v_{n}$. This reduces the computational cost of the method and simplifies the implementation.

From (2.5), the approximate solution of (1.5) is found by

$$
\begin{aligned}
u_{n}(x) & =\int_{D} k(x, y) v_{n}(y) d y+f(x) \\
& =\sum_{j=1}^{n} v_{n}\left(x_{j}\right) \int_{D} k(x, y) l_{j}(y) d y+f(x) .
\end{aligned}
$$

For the two approximate solutions, the following result holds:

Theorem 2.1. ([8, Theorem 2]) Assume conditions (A1) - (A4) hold and that the operator $P_{n}$ defined in (2.1) satisfies (2.2). Then

$$
\left\|v_{n}-v^{*}\right\| \rightarrow 0, \quad\left\|u_{n}-u^{*}\right\| \rightarrow 0, \text { as } n \rightarrow 0 .
$$

Moreover, there exists an $n_{0} \in \mathbb{N}$ and a constant $c$, independent of $n$, such that for all $n \geq n_{0}$,

$$
\left\|u_{n}-u^{*}\right\| \leq c \inf _{\phi \in D_{n}}\left\|\phi-v^{*}\right\|
$$

This means that $u_{n}$ converges to $u^{*}$ at least as fast as $v_{n}$ converges to $v^{*}$.

\subsection{Interpolation-based collocation}

To define the projection operator $P_{n}$, we start with piecewise linear interpolation of an unknown function on a triangle. First, we consider the unit simplex $\sigma=\{(s, t) \mid$ $0 \leq s, t, s+t \leq 1\}$. Let $h$ be a continuous function on $\sigma$ and denote by $w=1-s-t$. To approximate $h$, we use linear interpolation

$$
h(s, t) \approx \sum_{i=1}^{3} h\left(\tau_{i}\right) l_{i}(s, t)
$$

where the nodes

$$
\tau_{1}=\left(\frac{1}{2}, \frac{1}{2}\right), \tau_{2}=\left(\frac{1}{2}, 0\right), \tau_{3}=\left(0, \frac{1}{2}\right)
$$

are the midpoints of the three sides of $\sigma$ and

$$
l_{1}(s, t)=1-2 w, \quad l_{2}(s, t)=1-2 t, \quad l_{3}(s, t)=1-2 s
$$

are the corresponding Lagrange interpolation basis functions. Obviously, the approximation formula (2.7) is exact for all polynomials of degree less than or equal to 1.

This formula can be extended to any triangle $\Delta$, using an affine mapping $T$ : $\sigma \underset{\text { onto }}{\stackrel{1-1}{\longrightarrow}} \Delta$ given by

$$
x=(\xi, \eta)=T(s, t)=w z_{1}+t z_{2}+s z_{3},
$$

where $z_{1}, z_{2}, z_{3}$ are the vertices of $\Delta$. This mapping transforms a polynomial of degree $r$ in $(s, t)$ into a polynomial of the same degree in $(\xi, \eta)$ (and its inverse acts the same 
way on polynomials in $(\xi, \eta))$.

Denote by

$$
q_{i}=T\left(\tau_{i}\right), i=1,2,3 .
$$

For a given $h \in C(\Delta)$, define $P_{n} h$ by

$$
\begin{aligned}
P_{n} h(x) & =P_{n} h(T(s, t)) \\
& =\sum_{i=1}^{3} h\left(q_{i}\right) l_{i}(s, t), \quad(s, t) \in \sigma .
\end{aligned}
$$

Then the approximation formula

$$
h(x) \approx P_{n} h(x)=\sum_{i=1}^{3} h\left(q_{i}\right) l_{i}(s, t)
$$

is still exact for polynomials of degree $r \leq 1$.

From general interpolation theory, we have the following error bound for this approximation (see e.g. [2]).

Lemma 2.2. Let $\Delta$ be a planar triangle and assume $h \in C^{2}(\Delta)$. Then, the following holds

$$
\left\|h-P_{n} h\right\|_{\infty} \leq c \delta^{2}\left\|D^{2} h\right\|_{\infty},
$$

where $\delta=$ diameter $(\Delta)$ and $D^{2} h=\max _{0 \leq i \leq 2}\left|\frac{\partial^{2} h(\xi, \eta)}{\partial \xi^{i} \partial \eta^{2-i}}\right|$. The constant $c$ is independent of both $h$ and $\Delta$.

Now, to define our collocation method, let $\mathcal{T}_{n}=\left\{\Delta_{1}, \ldots, \Delta_{n}\right\}$ be a triangulation of $D$ with grid size $\delta_{n}$ and $T_{k}: \sigma \rightarrow \Delta_{k}$ be defined as in (2.10), for every $k=1, \ldots, n$. At each iteration, the triangulation is refined by splitting each triangle into four triangles, obtained by connecting the midpoints of the three sides. The new triangulation, $\mathcal{T}_{4 n}$, has four times as many triangles and grid size $\delta_{4 n}=\frac{1}{2} \delta_{n}$.

For a function $h \in C(D)$, restrict it to some $\Delta \in \mathcal{T}_{n}$ and use (2.13) to approximate it on $\Delta$.

Integrating (2.7), we obtain the quadrature formula

$$
\int_{\sigma} h(s, t) d \sigma \approx \frac{1}{6}\left[h\left(\frac{1}{2}, \frac{1}{2}\right)+h\left(\frac{1}{2}, 0\right)+h\left(0, \frac{1}{2}\right)\right] .
$$

Using the same affine mapping (2.10), this leads to a quadrature formula for integrals on $\Delta$

$$
\begin{aligned}
\int_{\Delta} h(y) d y & \approx \int_{\Delta} P_{n} h(y) d y \\
& =\sum_{i=1}^{3} h\left(q_{i}\right) \int_{\sigma} l_{i}(s, t) J_{T}(s, t) d \sigma
\end{aligned}
$$


where $J_{T}$ is the Jacobian of the transformation given in (2.10).

Thus, we have the approximation

$$
\begin{aligned}
\int_{D} h(y) d y & =\sum_{k=1}^{n} \int_{\Delta_{k}} h(y) d y \\
& \approx \sum_{k=1}^{n} \sum_{i=1}^{3} h\left(q_{k, i}\right) \int_{\sigma} l_{i}(s, t) J_{T_{k}}(s, t) d \sigma
\end{aligned}
$$

where $q_{k, i}=T_{k}\left(\tau_{i}\right), i=1,2,3$.

Then the collocation method is given by

$$
v_{n}\left(q_{i}\right)=g\left(q_{i}, \sum_{j=1}^{3} v_{n}\left(q_{j}\right) \int_{D} k\left(q_{i}, y\right) l_{j}(y) d y+f\left(q_{i}\right)\right),
$$

which leads to the system

$$
v_{n}\left(q_{i}\right)=g\left(q_{i}, \sum_{k=1}^{n} \sum_{j=1}^{3} v_{n}\left(q_{k, j}\right) \int_{\sigma} k\left(q_{i}, T_{k}(s, t)\right) l_{j}(s, t) J_{T_{k}}(s, t) d \sigma+f\left(q_{i}\right)\right),
$$

for all $i=1, \ldots, 3 n$. Once all the unknowns $v_{n}\left(q_{i}\right)$ are found from this system, we have, for each $x=T_{k}(s, t) \in \Delta_{k}$,

$$
\begin{aligned}
& v_{n}(x)=\sum_{i=1}^{3} v_{n}\left(q_{i}\right) l_{i}(s, t), \\
& u_{n}(x)=\sum_{i=1}^{3} v_{n}\left(q_{i}\right) \int_{\sigma} k\left(x, T_{k}(s, t)\right) l_{i}(s, t) J_{T_{k}}(s, t) d \sigma+f(x) .
\end{aligned}
$$

\section{Convergence and error analysis}

We write the system $(2.20)$ in operator form as

$$
\left(I-P_{n} \mathcal{K}\right) v_{n}=0
$$

with

$$
\mathcal{K}(v)(x)=g\left(x, \int_{D} k(x, y) v(y) d y+f(x)\right)
$$

and $P_{n}$ defined as in (2.12).

Since we are using piecewise linear interpolation in our collocation method, we have the following well known general result (see, e.g. [2, p. 177]).

Theorem 3.1. Assuming $\mathrm{A} 1-\mathrm{A} 4$ hold, the operators $I-P_{n} \mathcal{K}$ are invertible on $C(D)$ and have uniformly bounded inverses, for all sufficiently large $n$, say $n \geq n_{0}$. In addition, the following error bounds hold:

$$
\left\|v^{*}-v_{n}\right\|_{\infty} \leq\left\|\left(I-P_{n} \mathcal{K}\right)^{-1}\right\| \cdot\left\|v^{*}-P_{n} v^{*}\right\|, n \geq n_{0}
$$


and

$$
\left\|v^{*}-v_{n}\right\|_{\infty} \leq O\left(\delta^{2}\right), n \geq n_{0},
$$

where $\delta=\delta_{n}$ denotes the mesh size of the triangulation $\mathcal{T}_{n}$.

Now, this result holds in general, when using linear spline approximation. However, because of our particular choice of collocation (and interpolation) nodes, the approximation has higher order than $O\left(\delta^{2}\right)$. Notice that the quadrature formula $(2.15)$ has degree of precision $d=2$, higher than expected with interpolation of degree 1 . Then formula (2.16) also has degree of precision $d=2$. This will lead to a higher rate of convergence at the collocation nodes than $O\left(\delta^{2}\right)$, i. e., we get superconvergence.

Theorem 3.2. Assume the conditions $\mathrm{A} 1-\mathrm{A} 4$ hold, that $k \in C^{2}(D \times D)$ and that $v^{*} \in C^{3}(D)$. Then

$$
\max _{1 \leq i \leq n}\left|v^{*}\left(q_{i}\right)-v_{n}\left(q_{i}\right)\right|, \max _{1 \leq i \leq n}\left|u^{*}\left(q_{i}\right)-u_{n}\left(q_{i}\right)\right| \leq O\left(\delta^{3}\right) .
$$

Proof. The proof is computational and follows the same ideas as the ones given for similar results e.g. in $[3,10]$.

Since $v^{*}$ is the exact solution of $(1.4), v^{*}=\mathcal{K} v^{*}$. By the interpolation formula (2.13), $v_{n}=P_{n} \mathcal{K} v_{n}$. Then

$$
\begin{aligned}
\left(I-P_{n} \mathcal{K}\right)\left(v^{*}-v_{n}\right) & =v^{*}-v_{n}-P_{n} \mathcal{K} v^{*}+P_{n} \mathcal{K} v_{n} \\
& =\mathcal{K} v^{*}-P_{n} \mathcal{K} v^{*}=\left(I-P_{n}\right) \mathcal{K} v^{*}
\end{aligned}
$$

and, thus, under our assumptions, for each $i=1, \ldots, n$,

$$
\begin{aligned}
\left|\left(I-P_{n} \mathcal{K}\right)\left(v^{*}-v_{n}\right)\left(q_{i}\right)\right| & =\mid g\left(q_{i}, \int_{D} k\left(q_{i}, y\right) v^{*}(y) d y+f\left(q_{i}\right)\right) \\
& -g\left(q_{i}, \int_{D} k\left(q_{i}, y\right) P_{n} v^{*}(y) d y+f\left(q_{i}\right)\right) \mid \\
& \leq c\left|\sum_{k=1}^{n} \int_{\Delta_{k}} k\left(q_{i}, y\right)\left(I-P_{n}\right) v^{*}(y) d y\right|
\end{aligned}
$$

Now, on each triangle $\Delta_{k}$, let $p_{j}$ denote Taylor polynomial expansions of $v^{*}$ around a suitable point in $\Delta_{k}$, for $j=1,2$. Then

$$
\begin{aligned}
& \left\|v^{*}-p_{j}\right\|_{\infty} \leq c \delta^{j+1}, \\
& \left\|p_{2}-p_{1}\right\|_{\infty} \leq c \delta^{2} .
\end{aligned}
$$

Also, since $k \in C^{2}(D \times D)$, there exists a constant $k_{0}$ such that, for all $y \in \Delta_{k}$,

$$
\left|k\left(q_{i}, y\right)-k_{0}\right| \leq c \delta .
$$

Since the interpolation formula (2.13) has degree of precision 1, we have

$$
k\left(q_{i}, y\right)\left(I-P_{n}\right) p_{1}(y)=0
$$


and because the quadrature formula (2.15) has degree of precision 2, it follows that

$$
k_{0} \int_{\Delta_{k}}\left(I-P_{n}\right) p_{2}(y) d y=0 .
$$

Then, we can write

$$
\begin{aligned}
\left|\int_{\Delta_{k}} k\left(q_{i}, y\right)\left(I-P_{n}\right) v^{*}(y) d y\right| & \leq \mid \int_{\Delta_{k}} k\left(q_{i}, y\right)\left(I-P_{n}\right)\left(v^{*}-p_{2}\right)(y) d y \\
& +\int_{\Delta_{k}}\left(k\left(q_{i}, y\right)-k_{0}\right)\left(I-P_{n}\right)\left(p_{2}-p_{1}\right)(y) d y \\
& -\int_{\Delta_{k}} k_{0}\left(I-P_{n}\right) p_{1}(y) d y \mid .
\end{aligned}
$$

Now, using the bounds (3.5), (3.6), we get

$$
\begin{aligned}
\max _{1 \leq i \leq n}\left|v^{*}\left(q_{i}\right)-v_{n}\left(q_{i}\right)\right| & \leq \max _{1 \leq i \leq n}\left|\left(I-P_{n}\right)\left(\mathcal{K} v^{*}\right)\left(q_{i}\right)\right| \\
& \leq c \delta^{3} \sum_{k=1}^{n} \int_{\Delta_{k}} d y \\
& =O\left(\delta^{3}\right) \cdot n \cdot \operatorname{Area}\left(\Delta_{k}\right) \\
& =O\left(\delta^{3}\right) \cdot O\left(\delta^{-2}\right) \cdot O\left(\delta^{2}\right)=O\left(\delta^{3}\right) .
\end{aligned}
$$

Then by Theorem 2.1, we also have

$$
\max _{1 \leq i \leq n}\left|u^{*}\left(q_{i}\right)-u_{n}\left(q_{i}\right)\right| \leq O\left(\delta^{3}\right) .
$$

\section{Numerical experiments}

We will use the notation $x=\left(x_{1}, x_{2}\right), y=\left(y_{1}, y_{2}\right) \in D=[a, b] \times[c, d]$.

Example 4.1. Let us consider the integral equation

$$
u\left(x_{1}, x_{2}\right)=\frac{1}{2} \int_{0}^{1} \int_{0}^{1} \frac{x_{1}^{2}+1}{y_{1}^{2}+2 y_{2}^{2}} u^{2}\left(y_{1}, y_{2}\right) d y_{1} d y_{2}+2 x_{2}^{2}
$$

for $\left(x_{1}, x_{2}\right) \in[0,1] \times[0,1]$, with exact solution $u^{*}\left(x_{1}, x_{2}\right)=x_{1}^{2}+2 x_{2}^{2}+1$.

Here, we have

$$
\begin{aligned}
k(x, y) & =k\left(x_{1}, x_{2}, y_{1}, y_{2}\right)=\frac{x_{1}^{2}+1}{y_{1}^{2}+2 y_{2}^{2}} \\
g(y, u(y)) & =\frac{1}{2} u^{2}(y) \\
f(x) & =f\left(x_{1}, x_{2}\right)=2 x_{2}^{2}
\end{aligned}
$$


and $D=[0,1] \times[0,1]$.

We start with $n=2$ triangles that cover $D$ and then refine the triangulation as described earlier. We compute the errors

$$
\begin{aligned}
& e_{n}(v)=\max _{1 \leq i \leq n}\left|v^{*}\left(q_{i}\right)-v_{n}\left(q_{i}\right)\right| \text { and } \\
& e_{n}(u)=\max _{1 \leq i \leq n}\left|u^{*}\left(q_{i}\right)-u_{n}\left(q_{i}\right)\right|
\end{aligned}
$$

Also, we look at the ratios

$$
r_{1}=\frac{e_{n}(v)}{e_{4 n}(v)}, \quad r_{2}=\frac{e_{n}(u)}{e_{4 n}(u)}
$$

from one iteration to the next. If indeed the numerical method has order of convergence $O\left(\delta^{d}\right)$, then these ratios should equal approximately $2^{d}$.

We approximate the integrals needed for the coefficients of the nonlinear system $(2.20)$ using tiled adaptive quadratures (function integral2 in Matlab).

In Table 1 , we give the errors $e_{n}(v)$ and $e_{n}(u)$, as well as the values $\log _{2} r_{1}$ and $\log _{2} r_{2}$, for each iteration.

\begin{tabular}{ccccc}
\hline$n$ & $e_{n}(v)$ & $\log _{2} r_{1}$ & $e_{n}(u)$ & $\log _{2} r_{2}$ \\
\hline 2 & $1.121 e-1$ & & $2.382 e-2$ & \\
8 & $1.874 e-2$ & 2.58 & $3.397 e-3$ & 2.81 \\
32 & $2.582 e-3$ & 2.86 & $4.519 e-4$ & 2.91 \\
128 & $3.296 e-4$ & 2.97 & $5.653 e-5$ & 2.99 \\
\hline
\end{tabular}

TABLE 1. Errors in Example 4.1

The table shows that both $r_{1}$ and $r_{2}$ approach the value $2^{3}$, which is consistent with the superconvergence $O\left(\delta^{3}\right)$ proved in Theorem 3.2.

Example 4.2. Next, we consider the equation

$$
u\left(x_{1}, x_{2}\right)=\frac{1}{8} \int_{0}^{2} \int_{0}^{2} e^{2 x_{1}+x_{2}}\left(y_{1}^{2}-y_{2}\right) \ln \left(u\left(y_{1}, y_{2}\right)\right) d y_{1} d y_{2},
$$

for $\left(x_{1}, x_{2}\right) \in[0,2] \times[0,2]$, whose exact solution is $u^{*}\left(x_{1}, x_{2}\right)=e^{2 x_{1}+x_{2}}$.

In this case, $D=[0,2] \times[0,2]$ and we can take

$$
\begin{aligned}
k(x, y) & =\frac{1}{8} e^{2 x_{1}+x_{2}}, \\
g(y, u(y)) & =\ln (u(y)), \\
f(x) & =0 .
\end{aligned}
$$

Again, we start with $n=2$ triangles and proceed as before.

The errors are shown in Table 2 . The results show, again, an $O\left(\delta^{3}\right)$ rate of convergence. 


\begin{tabular}{ccccc}
\hline$n$ & $e_{n}(v)$ & $\log _{2} r_{1}$ & $e_{n}(u)$ & $\log _{2} r_{2}$ \\
\hline 2 & $2.143 e-1$ & & $3.628 e-2$ & \\
8 & $3.344 e-2$ & 2.68 & $5.356 e-3$ & 2.76 \\
32 & $4.735 e-3$ & 2.82 & $7.428 e-4$ & 2.85 \\
128 & $6.086 e-4$ & 2.96 & $9.415 e-5$ & 2.98 \\
\hline
\end{tabular}

TABLE 2. Errors in Example 4.2

\section{Conclusions}

We have developed a collocation method for the approximate solution of nonlinear Hammerstein integral equations over bounded domains of $\mathbb{R}^{2}$. The numerical scheme is based on a special choice of linear spline interpolation over triangles. Having a higher degree of precision of the quadrature formula than expected, the resulting collocation method is superconvergent at the collocation nodes, converging faster than over the entire domain. This is one major advantage of the proposed numerical scheme over other projection-type collocation methods. Another important aspect is the fact that by applying collocation to a newly reformulated integral equation, the integrals needed for the coefficients of the linear system only have to be evaluated once, not at every iteration, which reduces the computational cost of the method and simplifies the implementation. Thus, this is a very efficient numerical method.

These ideas can be taken further, considering more complicated domains, higher dimensions or other types of interpolation.

\section{References}

[1] Abdolmaleki, E., Saberi Najafi, H., An efficient algorithmic method to solve Hammerstein integral equations and application to a functional differential equation, Adv. Mech. Eng., 9(2017), no. 6, 1-8.

[2] Atkinson, K.E., The Numerical Solution of Integral Equations of the Second Kind, Cambridge Univ. Press, 1997.

[3] Atkinson, K.E., Chandler, G., The collocation method for solving the radiosity equation for unoccluded surfaces, J. Integral Eqs. Appl., 10(1998), 253-290.

[4] Bakodah H., Darwish, M., On discrete adomian decomposition method with Chebyshev abscissa for nonlinear integral equations of Hammerstein type, Adv. Pure Math., 2(2012), no. $5,310-313$.

[5] Dastjerdi, H.L., Ghaini, F.M.M., The discrete collocation method for FredholmHammerstein integral equations based on moving least squares method, Int. J. Comput. Math., 93(2016), no. 8, 1347-1357.

[6] Gnecco, G., Kůrková, V., Sanguineti, M., Accuracy of approximations of solutions to Fredholm equations by kernel methods, Appl. Math. Comp., 218(2012), 7481-7497.

[7] Hashemizadeh, E., Rostami, M., Numerical solution of Hammerstein integral equations of mixed type using the Sinc-collocation method, J. Comput. Appl. Math., 279(2015), 31-39. 
[8] Kumar, S., Sloan, I.H., A new collocation-type method for Hammerstein integral equations, Math. of Comp., 48(1987), 585-593.

[9] Mastroianni, G., Milovanović, G.V., Occorsio, D., Nyström method for Fredholm integral equations of the second kind in two variables on a triangle, Appl. Math. Comp., 219(14)(2013), 7653-7662.

[10] Micula, S., A spline collocation method for Fredholm-Hammerstein integral equations of the second kind in two variables, Appl. Math. Comp., 265(2015), 352-357.

[11] Rahimkhani, P., Ordokhani, Y., Numerical solution of Volterra-Hammerstein delay integral equations, Iran J. Sci. Technol. Trans. Sci., 44(2020), 445-457.

[12] Sahu, P.K., Ranjan, A.K., Saha Ray, S., B-spline wavelet method for solving FredholmHammerstein integral equation arising from chemical reactor theory, Nonlin. Eng., 7(2018), no. 3, 163-169.

[13] Wazwaz, A.M., Linear and Nonlinear Integral Equations, Methods and Applications, Higher Education Press, Beijing, Springer Verlag, New York, 2011.

Sanda Micula

Babeş-Bolyai University,

Faculty of Mathematics and Computer Sciences,

1, Kogălniceanu Street,

400084 Cluj-Napoca, Romania

e-mail: smicula@math.ubbcluj.ro 